\title{
Trastornos alimentarios del lactante y preescolar
}

\author{
PAULINA BRAVO J. ${ }^{1}$, M. ISABEL HODGSON B. ${ }^{1}$ \\ 1. Unidad de Gastroenterología y Nutrición Pediátrica, División de Pediatría, Facultad de Medicina, \\ Pontificia Universidad Católica de Chile.
}

\begin{abstract}
\section{Eating disorders in nursing and preschool children}

Eating disorders among nursing and preschool children are a common cause of outpatient consultation in pediatrics and nutrition. It is essential to rule out organic causes, especially when there is nutritional involvement. Among non-organic causes there are several subtypes depending upon the trigger. Environmental and nutritional factors can be modified. Curiously, many of them are directly related to the perception of hunger and satiety the individual will develop from childhood.
\end{abstract}

(Key words: Eating disorders, hunger, satiety).

Rev Chil Pediatr 2011; 82 (2): 87-92

\section{RESUMEN}

Los trastornos de la alimentación en el lactante y preescolar siguen siendo un motivo frecuente de consulta en policlínicos de pediatría y nutrición infantil. Descartar causas orgánicas, sobre todo cuando existe compromiso nutricional, es de fundamental importancia. Dentro de los trastornos de origen no orgánico existen varios subtipos dependiendo de la causa desencadenante. Muchos factores involucrados tanto ambientales como nutricionales, son susceptibles de ser modificados. Curiosamente, muchos de ellos se relacionan directamente con la capacidad de percepción de las sensaciones de hambre y saciedad que el individuo desarrollará desde niño. (Palabras clave: desordenes de la alimentación, hambre, saciedad).

Rev Chil Pediatr 2011; 82 (2): 87-92

Trabajo recibido el 04 de abril de 2011, devuelto para corregir el 14 de abril de 2011, aceptado para publicación el 29 de abril de 2011.

Correspondencia a:

Dra. Paulina Bravo J.

E-mail: pbravoj@med.puc.cl 


\section{Introducción}

Pese a las alarmantes cifras de obesidad en niños chilenos, siendo éstas de $10,6 \%$ en niños en el grupo etario de 2 a 5 años ${ }^{1}$, el nivel de consulta de lactantes y preescolares con dificultades para alimentarse parece permanecer estable tanto en policlínicos de nutrición infantil como en los de pediatría general. La exacta incidencia de este heterogéneo problema es difícil de precisar en nuestro medio y a nivel mundial, debido a que no se cuenta con una clara definición del problema. Se estima que hasta un $25 \%$ de niños de otra manera sanos y un $80 \%$ de aquellos con necesidades especiales, sufren de algún trastorno de la alimentación infantil ${ }^{2}$, ocurriendo con mayor frecuencia en el rango de edad entre los 7 y 11 meses $^{4,5}$, probablemente por coincidir con el inicio y establecimiento de la alimentación complementaria. Es así como podemos encontrar a estos pacientes bajo el nombre de "anorexia infantil", "trastorno alimentario" o "trastorno del apetito”. Siendo éste frecuentemente descrito en el mundo anglosajón como "picky eater", definido como la presencia de alguna de las siguientes conductas: 1) No come suficiente; 2) Frecuentemente es muy selectivo; 3) Usualmente come muy lento².

En 1994 el Manual Diagnóstico y Estadís- tico de Enfermedades Mentales (DSM-IV) ${ }^{3}$ introdujo el término: "Trastornos de la alimentación”, definiéndolo como un disturbio para alimentarse manifestado por una persistente dificultad para comer adecuadamente, asociado a dificultad para ganar peso o la pérdida de peso significativa por al menos un mes, que se inicia antes de los 6 años de edad. Por otro lado, Chatoor $^{4}$ junto a un grupo de expertos propuso una clasificación con seis subtipos de este trastorno, los cuales no necesariamente son excluyentes y que se describen en la tabla 1 . Recientemente, Levy y cols ${ }^{6}$, realizaron un análisis discriminativo entre 21 variables clínicas, evaluando tanto a niños menores de seis años, con síntomas y signos de presentación antes de los dos años, como a sus padres, con el objeto de discriminar con mayor precisión aquellos trastornos alimentarios de origen conductual. Es así como los Criterios de Wolfson demostraron tener un $98 \%$ de sensibilidad para determinar el origen conductual del trastorno, habiéndose usado los criterios de DSM-IV como la referencia estándar. Estos criterios comprenden: 1) Rechazo alimentario por más de un mes; 2) Ausencia de enfermedad orgánica; 3) Edad de comienzo de algunos signos o síntomas antes de los 2 años y edad de presentación antes de los seis años; 4) Presencia de alimentación patológica y/o vómitos anticipatorios. Algu-

Tabla 1. Tipos de desórdenes alimentarios del lactante y preescolar según Chatoor y cols ${ }^{4}$

\begin{tabular}{|c|c|}
\hline Tipo de desorden & Descripción abreviada \\
\hline Desorden alimentario del estado de regulación & $\begin{array}{l}\text { Inicio durante los primeros meses de vida, dificultad para mantener calma } \\
\text { o alerta en períodos en que es alimentado. Falla para ganar peso. Orga- } \\
\text { nicidad descartada }\end{array}$ \\
\hline Desorden alimentario de la reciprocidad cuidador-niño & $\begin{array}{l}\text { Usualmente durante el primer año de vida. Falla de medro. Carencia de } \\
\text { contacto visual o sonrisa con sus padres durante la alimentación }\end{array}$ \\
\hline Anorexia infantil & $\begin{array}{l}\text { Usualmente niño(a) entre } 6 \text { meses y } 3 \text { años de edad que rechaza comer } \\
\text { cantidades adecuadas. Frecuentemente durante la transición hacia alimen- } \\
\text { tación independiente. Con desbordante energía, no tiene percepción de } \\
\text { hambre. Falla de medro }\end{array}$ \\
\hline Aversión sensorial & $\begin{array}{l}\text { Consistente rechazo a ciertos alimentos, relacionado con textura, tempe- } \\
\text { raturas y olores. Neofobia (rechazo a alimentos nuevos) }\end{array}$ \\
\hline Desorden alimentario postraumático & $\begin{array}{l}\text { Comienzo agudo de rechazo alimentario severo y consistente que sigue a } \\
\text { un evento o repetidos insultos traumáticos. Puede ocurrir a cualquier edad }\end{array}$ \\
\hline Desorden alimentario asociado a comorbilidades & $\begin{array}{l}\text { Existe una condición médica concurrente que explica el rechazo alimen- } \\
\text { tario }\end{array}$ \\
\hline
\end{tabular}


nos ejemplos de alimentación patológica son: Alimentación nocturna, multiples intentos, en lugares o horarios inhabituales por parte de los padres para alimentar al niño, alimentación forzada, alimentación mecanizada, alimentación con distracción, entre otros. Todas las anteriores representan estrategias desesperadas del adulto que alimenta al niño pero que lamentablemente, no respetan la sensación de hambre o saciedad del infante. La tardía identificación y la carencia de un adecuado manejo de esta entidad puede provocar desde estudios invasivos innecesarios hasta la instalación de un tubo de gastrostomía, así como consecuencias psicológicas complejas como por ejemplo, conductas aversivas secundarias a patrones de alimentación disfuncional por parte de los padres.

\section{Factores etiológicos involucrados}

Los trastornos alimentarios no tienen una sola etiología determinada, al hacer una evaluación detallada se pueden encontrar involucrados factores genéticos, ambientales, conductuales, emocionales y orgánicos (gastrointestinal, neurológico, cardiológico, respiratorio, endocrinológico, nefrourológico, psiquiátrico, entre otros).

La persistencia de estos trastornos y su coexistencia con altas cifras de malnutrición por exceso en la población infantil, sugiere que nuestro actual estilo de vida está influyendo en el comportamiento de nuestros niños y en su relación con la comida, teniendo responsabilidades tanto en la avidez como en el rechazo a ésta. La rápida transición nutricional chilena que dejó atrás las altas cifras de desnutrición infantil en los años 70, llevándonos hacia un fuerte predominio del sobrepeso y obesidad desde la década del noventa, puede explicar en parte este fenómeno ${ }^{6}$. Hoy en día nos enfrentamos diariamente a una mayor oferta de alimentos de menor valor nutricional y alta densidad energética. Asociado a lo anterior, el acelerado estilo de vida actual, con cada vez menos tiempo para la preparación de comidas caseras, el compartir las comidas en familia y modelar conductas alimentarias saludables son factores importantes de considerar. En este contexto, el factor ambiental parece contribuir prácticamente en todos los subtipos de estos trastornos en diferente proporción. Varios factores en el desarrollo del niño tienden a converger en la mayor o menor conexión que éste tiene con las sensaciones de hambre y saciedad. Afortunadamente estos factores son susceptibles de modificar a lo largo de la vida del niño (en la figura 1 se pueden observar graficados algunos de ellos).

Este artículo pretende revisar estrategias que pueden contribuir a evitar una disfunción en el proceso de ser alimentado y de alimentarse, sin abordar los trastornos de alimentación de origen orgánico por constituir un grupo muy heterogéneo de afecciones, que requieren de un enfoque específico, que se escapa del alcance del presente texto. Entre los factores ambientales, estructurales y nutricionales susceptibles de modificar hay que considerar los siguientes:

\section{Restricciones dietarias de la madre durante la lactancia}

Las características positivas de la leche humana son universalmente conocidas. Una de las menos difundidas es la capacidad de traspasar al feto una variedad de sabores derivados de los alimentos consumidos por su madre ${ }^{7}$. Esta cualidad refuerza la superioridad de la leche humana frente a formulas lácteas, sobre todo considerando el monótono sabor de estas últimas. En consecuencia la leche humana proporciona a los lactantes una oportunidad de aprender a degustar variados sabores, lo cual facilitará la introducción de nuevos alimentos durante el segundo semestre de la vida ${ }^{8}$. Por lo anterior, evitar la múltiples restricciones alimentarias a la nodriza podría teóricamente mejorar la aceptabilidad de nuevos alimentos durante la infancia.

\section{Adecuada transición a la alimentación complementaria}

Es sabido que lactantes expuestos a una mayor variedad de alimentos aceptan más rápidamente nuevas comidas, incluso aquellos alimentos frecuentemente rechazados en los inicios, como los vegetales verdes, son acep- 


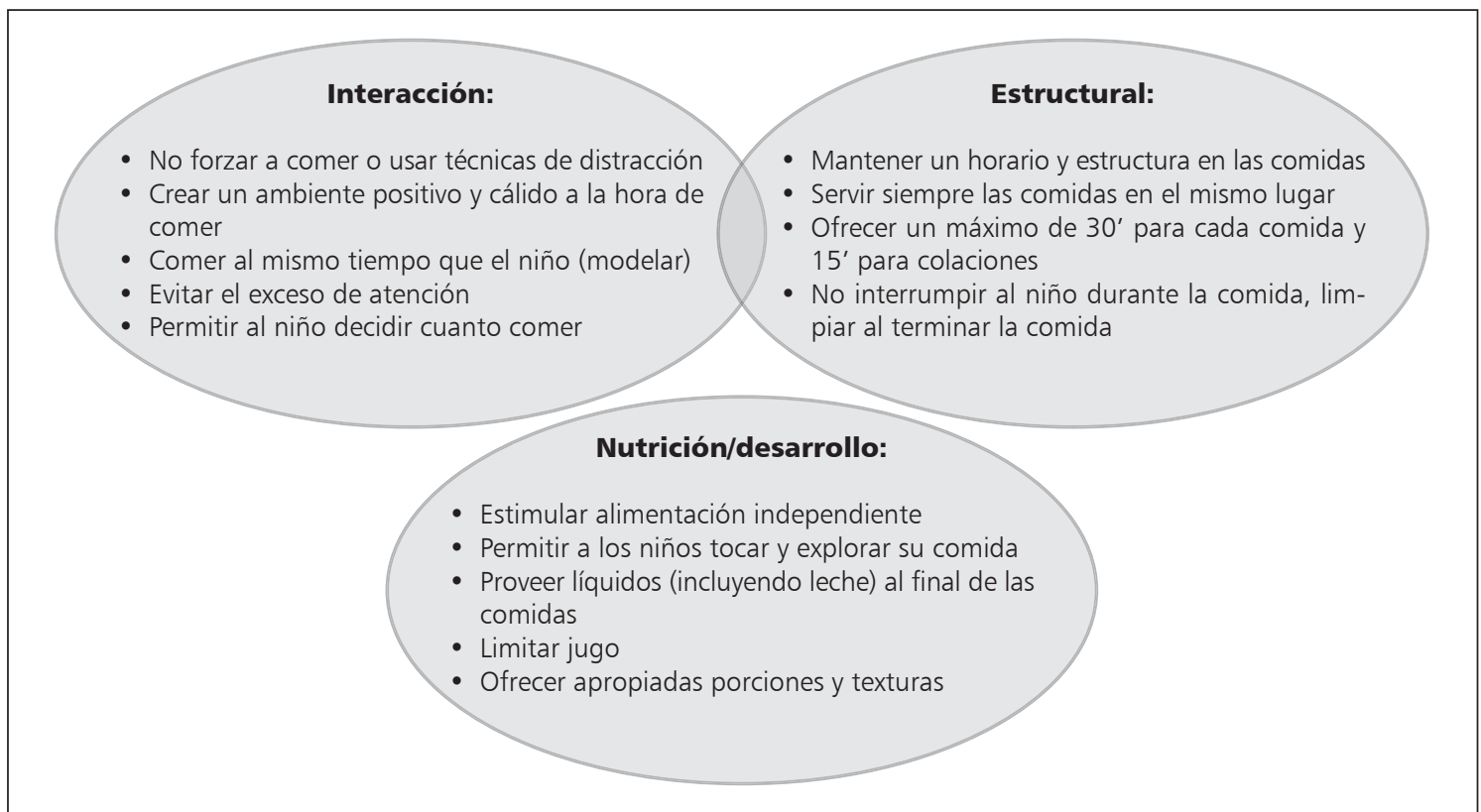

Figura 1. Distintos ámbitos a evaluar y posibles estrategias propuestas en el manejo de lactantes y preescolares con trastornos de alimentación.

tados después de una exposición repetida ${ }^{9}$. Por otro lado, la textura de los alimentos empieza a ser un problema al intentar cambiar la consistencia tipo puré por comidas con doble consistencia, siendo éste el momento de mayor dificultad pues el niño no estará preparado para manejar dos o más consistencias dentro de un medio líquido en forma eficiente, provocando muchas veces la perpetuación de los purés hasta edad avanzada. Varias de nuestras comidas típicas son preparadas mezclando múltiples alimentos de distintas texturas y sabores en un solo plato y en un medio líquido, lo que suele causar confusión y rechazo en el niño. En general, los niños pequeños responderán mejor ante la presentación de alimentos claramente individualizados en el plato.

\section{3. "Finger foods"}

El uso de alimentos y sus presentaciones de manera tal que sean fáciles de explorar y manipular por el niño, favorece la aceptación de una mayor variedad de alimentos además de fomentar la autoalimentación precoz. $\mathrm{La}$ introducción de diferentes alimentos de todos los grupos alimentarios, de textura suave, fá- ciles de masticar o bien crujientes y disolvibles, estimulan la alimentación independiente y ayudan al niño a conectarse precozmente con su percepción de hambre y saciedad. Muchas veces pretendemos pasar bruscamente desde la fase en la cual el niño es alimentado pasivamente a, la del uso de los utensilios, sin permitirle tocar o manipular los alimentos, con la consiguiente dificultad, frustrando tanto al padre como al niño, y regresando muchas veces a la etapa del puré.

\section{Elección de texturas adecuadas}

La textura de los alimentos presentados a lactantes y preescolares debiera ser cuidadosamente graduada de acuerdo al estado de desarrollo motriz oral de cada niño. Habitualmente a la edad de 7-8 meses los movimientos verticales de la mandíbula y lengua (arriba/abajo) son los predominantes. Alrededor de los 12 meses se adquieren movimientos rotatorios muy rudimentarios, siendo el niño aún ineficiente en los movimientos de lateralización de la comida con su lengua. Recién entre los 15 y 18 meses el niño logra una masticación rotatoria madura, con movimientos vertica- 
les, horizontales y oblicuos. Antes de esto, es recomendable pedir a los padres que prueben masticar sólo con movimientos verticales de la mandíbula cada alimento previo a ofrecérselo al niño con el objeto de testear si dicho alimento es adecuado para la etapa de desarrollo en que se encuentra el niño ${ }^{10,11}$. Además del riesgo de asfixia (chocking) que tiene el uso de texturas inadecuadas, el niño puede frustrarse, cansarse y rechazar nuevos alimentos. Por otro lado, el mantener texturas tipo puré más allá de la etapa recomendable puede alterar el normal desarrollo motor oral.

\section{La influencia de los padres, cuidadores y pares}

Estudios observacionales han mostrado que los niños aprenden a aceptar ciertos alimentos por observación directa de personas cercanas. El rol modelador de la familia es clave ${ }^{12}$. Actualmente, es cada vez menos frecuente que el lactante y/o preescolar participe diariamente en la mesa familiar, lugar en donde debiera tener la oportunidad de interactuar con sus padres y/o hermanos y de adquirir hábitos saludables. Sánchez y cols ${ }^{13}$, analizaron 34 casos de trastornos de alimentación de origen conductual, provenientes del servicio de salud público y privado de Santiago, observando que éstos fueron más frecuentemente alimentados en forma separada del grupo familiar, comparados con un grupo control $(\mathrm{p}=0,01)$.

Así mismo, la influencia de otros cuidadores como educadores y asesoras domésticas también tiene un rol importante como modelo. Hughes y cols ${ }^{14}$, mostraron la influencia en el desarrollo de conductas saludables y no saludables que ejercieron 50 educadores de jardines infantiles sobre 549 niños en EEUU.

\section{Limitar calorías líquidas}

Cerca de los 6 meses el lactante debiera iniciar una transición desde un 100\% de la ingestión de calorías y nutrientes provenientes de la leche humana o en su defecto fórmula, a una dieta sólida, la cual aportará nutrientes complementarios. Especial énfasis dentro de estos nutrientes merece el fierro, considerando la disminución de sus depósitos a partir del segundo semestre de la vida. Desafortunadamen- te, esta adecuada transición no siempre se realiza de manera óptima. Muchos niños mayores de un año continúan recibiendo altos volúmenes de leche, lo cual podría suprimir el apetito para una dieta sólida más variada. Asociado a esto, el consumo de jugos en altas cantidades aporta al lactante o preescolar un exceso de azúcares con escaso contenido nutricional. La recomendación es adecuar el volumen de leche de acuerdo a los requerimientos de cada edad, limitar el consumo de jugo al mínimo (tendiendo a cero) y ofrecer los líquidos al final de cada comida con el objeto de no suprimir el apetito para alimentos de mayor calidad nutritiva.

\section{Fomentar la alimentación independiente}

Ciertos estilos parentales que tienden a regular y controlar la alimentación de sus hijos sin responder a señales de hambre y saciedad, han sido asociados positivamente con dificultades para regular ingesta energética ${ }^{15}$. Así mismo, Carruth y cols ${ }^{16}$, a través de encuestas telefónicas a más de 3000 familias de preescolares, demostraron que los niños que lograban una alimentación independiente a menor edad, dentro del segundo año de vida, tenían una mayor ingesta de nutrientes que aquellos que eran alimentados pasivamente. Por esto es altamente recomendable permitir al niño explorar sus alimentos y facilitarle una dieta fácil de ser manipulada, ofrecerle una segunda cuchara mientras es alimentado y en un siguiente paso, considerar el primer período de la comida para que el niño explore e intente comer por sí mismo, para finalmente sólo ser supervisado a las horas de comida.

\section{Considerar aversión sensorial dentro del diagnóstico diferencial}

El trastorno alimentario tipo aversión sensorial cuyos criterios diagnósticos han sido publicados por Chatoor ${ }^{4}$, también conocido como Desorden de la Integración Sensorial, debe ser considerado dentro del diagnóstico diferencial del niño con trastornos de la alimentación, en especial cuando existe un persistente rechazo de alimentos con ciertos sabores, texturas, temperaturas u olores en particular. Existen cuestionarios de pesquisa para evaluar diferentes aspectos de hiperreactividad a estímulos 
táctiles (molestia con etiquetas de la ropa, jugar con plastilina o arena), auditivos (molestia con ruidos específicos), visuales (se disgusta en lugares demasiado luminosos) y olfativos (no tolera ciertos olores), los cuales pueden dar las claves para este diagnóstico. Su manejo requiere un equipo multidisciplinario, con especial énfasis en terapeutas ocupacionales y fonoaudiólogos con formación en esta área. Su manejo debe ser orientado a un trabajo intensivo utilizando técnicas de desensibilización, entre muchas otras estrategias.

\section{Conclusión}

Aún cuando la exacta prevalencia de los trastornos de alimentación del lactante y preescolar es muy difícil de estimar debido a la carencia actual de consenso en cuanto a su definición; estos parecen mantenerse como una causa frecuente de consulta pediátrica y nutricional.

Muchos factores, tanto ambientales como nutricionales, involucrados en la génesis de estos trastornos son susceptibles de modificar. Es tarea de los pediatras educar y guiar a los padres y cuidadores en las distintas etapas del desarrollo, a fin de lograr establecer un patrón óptimo de alimentación, que se traduzca en una relación sana y natural con la comida, reconociendo y respetando las sensaciones fisiológicas de hambre y saciedad.

\section{Referencias}

1.- Chile. Junta Nacional de Auxilio Escolar y Becas de Chile (JUNAEB) http://www.junaeb.cl

2.- Chatoor I, Ganabian J: Food refusal by infants and young children: diagnosis and treatment. Cogn Behav Pract 2003; 10: 138-46.

3.- Marchi, $M$, Cohen P: Early childhood eating behaviors and adolescent eating disorders. Journal of the American Academy of Child and Adolescent Psychiatry 2003; 42 (1): 76-84.

4.- American Psychiatric Association: (1994). Diagnostic and statistical manual of mental disorders ( $4^{\text {th }} \mathrm{Ed}$.).
Washington, DC: Author.

5.- Chatoor I: Diagnosis and treatment of feeding disorders in infants, toddlers and young children. 2009. Washington, DC: ZERO TO THREE.

6.- Levy Y, Levy A, Zangen T, et al: Journal of Pediatric Gastroenterology and Nutrition 2009; 48: 355-62.

7.- Carruth BR, Ziegler PJ, Gordon A, Barr SI: Prevalence of picky eater among infants and toddlers and their caregivers; decisions about offering a new food. Journal of the American Dietetic Association 2004; 104 (S1): S57-S64.

8.- Vio F, Albala C: La transición nutricional en Chile. Rev Chil Nutr 1998; 25: 11-20.

9.- Mennella JA, Beauchamp GK: Maternal diet alters the sensory qualities of human milk and the nursling's behavior. Pediatrics 1991; 88 (4): 737-44.

10.- Mennella JA, Jagnow CP, Beauchamp GK: Prenatal and postnatal flavor learning by human infants. Pediatrics 2001; 107 (6): e88.

11.- Maier A, Chabanet C, Schaal B, Issanchou S, Leathwood P: Effects of repeated exposure on acceptance of initially disliked vegetables in 7-month old infants. Food Quality and Preference 2007; 18 (8): 1023-32.

12.- Evans Morris S, Dunn Klein M: Pre-Feeding Skills, Second Edition. Therapy Skill Builders, 2000.

13.- Stolvitz P, Gisel EG: Circumoral movements in response to three different food textures in children 6 months to 2 years of age. Dysphagia 1991; 6 (1): 17-25.

14.- Young EM, Fors SW, Hayes DM: Association between perceived parent behaviors and middle school student fruits and vegetable consumption. J Nutr Educ Behav 2004; 36 (1): 2-8.

15.- Birch Ll: Effects of peers' models food choices and eating behaviors on preschooler' food preferences. Child Development 1980; 51: 489-96.

16.- Hughes $O$, Patrick H, Power TG, et al: Impact of Child Care Providers' Feeding on Children's Food Consumption Journal of Developmental \& Behavioral Pediatrics 2007; 28: 100-7.

17.- Birch $L L$, Davison KK: Family environmental factors influencing the developing behavioral controls of food intake and childhood overweight. Pediatr Clin North Am 2001; 48: 893-907.

18.- Carruth BR, Ziegler PJ, Gordon A, Hendricks K: Developmental Milestones and Self-Feeding. Behaviors in Infants and Toddlers. J Am Diet Assoc 2004; 104 (1 Suppl 1): s51-6. 